Pacific Journal of 


\title{
MULTIPLY TRANSITIVE GROUPS OF TRANSFORMATIONS
}

\author{
JAMES V. WHITTAKER
}

\begin{abstract}
A group $G$ of homeomorphisms of a topological space $X$ onto itself is called $n$-transitive if any set of $n$ points in $X$ can be mapped onto any other set of $n$ points by some member of $G$. In this paper, we investigate the transitivity of $G$ when $X$ is euclidean $m$-space $E^{m}$ or real projective $m$-space $I^{m}$, and $G$ properly contains the group $A_{m}$ of affine transformations or the group $P_{m}$ of projective transformations, respectively. We show that $G \supset A_{1}$ implies that $G$ is at least 3-transitive, $G \supset P_{1}$ implies that $G$ is at least 4-transitive, and, for a fairly wide class of groups, $G$ is $n$-transitive for every $n$. For higher dimensional spaces, our information is considerably more meager. We show that $G \supset A_{m}$ or $G \supset P_{m}$ implies that $G$ is at least 3 -transitive, and that if some member of $G$ leaves fixed the points of some open set, then $G$ is $n$-transitive for every $n$.
\end{abstract}

2. Multiple transitivity. Let $X$ be a topological space and $H(X)$ the group of all homeomorphisms of $X$ onto itself. The identity of $H(X)$ will be denoted by $e$. For each $h \in H(X)$, we set $K(h)=$ $\{x \in X: h(x)=x\}$, and observe that

$$
K\left(h_{1} h_{2}\right) \supset K\left(h_{1}\right) \cap K\left(h_{2}\right), \quad K\left(h_{1} h_{2} h_{1}^{-1}\right)=h_{1}\left(K\left(h_{2}\right)\right) .
$$

For any subgroup $G$ of $H(X)$ and any $x \in X$, we call $G(x)=\{g(x): g \in G\}$ an orbit of $G$ and note that orbits are either coincident or disjoint. When $n$ is a positive integer, we define $G$ to be $n$-transitive if, for any subsets $\left\{x_{1}, \cdots, x_{n}\right\},\left\{y_{1}, \cdots, y_{n}\right\}$ of $n$ distinct points in $X$, we can find $g \in G$ such that $g\left(x_{i}\right)=y_{i}(i=1, \cdots, n)$. If $g$ is unique, we call $G$ strictly $n$-transitive. If $G$ is $n$-transitive for every $n$, we will call $G \omega$-transitive. When $X$ is a connected, locally euclidean manifold of dimension $m \geqq 2$, then $H(X)$ is clearly $\omega$-transitive, but $H\left(E^{1}\right)$ is only 2-transitive, and $H\left(\Pi^{1}\right)$ is only 3-transitive under the above definition. To remedy this, we will modify the definition in these two cases by requiring that as $i$ increases from 1 to $n, x_{i}$ should move in the positive sense of orientation, and $y_{i}$ should move in either the positive or negative sense. Thus $H(X)$ is also $\omega$-transitive when $X=E^{1}$ or $\Pi^{1}$. The group $H^{+}(X)$ of orientation-preserving homeomorphisms of $X$ evidently sends any positively oriented $n$-tuple into any other positively oriented $n$-tuple for every $n$. We will say that a subgroup $G$ of $H^{+}(X)$ is $n$-transitive relative to $H^{+}(X)$ if $G$ sends any positively oriented $n$-tuple into any other positively oriented $n$-tuple. 
Lemma 1. Let $X$ be a topological space and $G$ a subgroup of $H(X)$. Suppose that, for each subset $L$ of $n$ points in $X$ and each $x \in X-L$, the orbit $G_{0}(x)$ of the group $G_{0}=\{g \in G: L \subset K(g)\}$ has a nonempty interior in $X$. Then $G_{0}(x)$ contains a connected component of $X-L$.

Proof. Let $U \subset G_{0}(x)$ be an open subset of $X$, and $y \in G_{0}(x)$ be arbitrary. Then we can find $g_{1}, g_{2} \in G_{0}$ with the properties $g_{1}(x) \in U$ and $g_{2}(x)=y$. Thus $y=g_{2}(x) \in g_{2} g_{1}^{-1}(U) \subset G_{0}(x)$, and $y$ lies in the interior of $G_{0}(x)$, so that $G_{0}(x)$ is open. The orbits of $G_{0}$ are either coincident or disjoint, and no two of them can intersect the same connected component of $X-L$ unless they coincide. Since $e \in G_{0}$, we have $x \in G_{0}(x)$, and the orbits $G_{0}(x)$ cover $X-L$. Hence, each of them contains a connected component.

Lemma 2. With the same hypotheses as in Lemma 1, suppose $X$ is a connected, locally euclidean manifold of dimension $m \geqq 2$, and $G$ is n-transitive for some $n$. Then $G$ is $(n+1)$-transitive.

Proof. To show that $G$ is $(n+1)$-transitive, it is evidently sufficient to show that, for any points $x_{1}, \cdots, x_{n+1}, y_{n+1} \in X$, there is a $g \in G$ satisfying $g\left(x_{i}\right)=x_{i} \quad(i=1, \cdots, n)$ and $g\left(x_{n+1}\right)=y_{n+1}$. Since $X-\left\{x_{1}, \cdots, x_{n}\right\}$ is connected, this is precisely the conclusion of Lemma 1.

Lemma 3. With the same hypotheses as in Lemma 1, suppose $X=E^{1}, G$ is n-transitive for some $n \geqq 2$, and the condition " $x \in X-L$ " is replaced by " $x$ lies to the right of $L$ ". Then $G$ is $(n+1)$-transitive. If $G \subset H^{+}\left(E^{1}\right)$ is n-transitive $(n \geqq 0)$ relative to $H^{+}\left(E^{1}\right)$, then $G$ is $(n+1)$-transitive relative to $H^{+}\left(E^{1}\right)$.

Proof. Let $x_{1}<\cdots<x_{n+1}$ and either (i) $y_{1}<\cdots<y_{n+1}$ or (ii) $y_{1}>\cdots>y_{n+1}$ be given. In case (i), we choose $g_{1} \in G$ so that $g_{1}\left(x_{i}\right)=y_{i}$ $(i=1, \cdots, n)$. Since $g_{1}$ is order-preserving, we have $g_{1}\left(x_{n+1}\right)>y_{n}$, and the same argument as in the proof of Lemma 1 shows that the orbit $G_{0}\left(g_{1}\left(x_{n+1}\right)\right)$ is the open interval $\left(y_{n}, \infty\right)$, where $G_{0}=\{g \in G$ : $\left.\left\{y_{1}, \cdots, y_{n}\right\} \subset K(g)\right\}$. Thus we can find $g_{2} \in G_{0}$ satisfying $g_{2}\left(g_{1}\left(x_{n+1}\right)\right)=$ $y_{n+1}$, so that $g_{2} g_{1}\left(x_{i}\right)=y_{i}(i=1, \cdots, n+1)$. This also suffices to prove the last statement in the Lemma. In case (ii), we choose $g_{3} \in G$ so that $g_{3}\left(x_{i}\right)=y_{i}(i=2, \cdots, n+1)$. From $n \geqq 2$ we infer that $g_{3}$ is order-reversing, whence $g_{3}\left(x_{1}\right)>y_{2}$, and we can find $g_{4} \in G$ satisfying $y_{i} \in K\left(g_{4}\right)(i=2, \cdots, n+1)$ and $g_{2}\left(g_{3}\left(x_{1}\right)\right)=y_{1}$. Thus $g_{4} g_{3}\left(x_{i}\right)=y_{i}$ $(i=1, \cdots, n+1)$. 
If, in the hypothesis of Lemma 3 , " $x$ lies to the right of $L$ " is replaced by " $x$ lies to the left of $L$ ", then an argument similar to the preceding one yields the same conclusions.

3. Extensions of finite sets. Let $L$ be a finite subset of an arbitrary subset $M$ of a topological space $X$, and $G$ a subgroup of $H(X)$. We set $M_{0}=M$ and, for $i \geqq 0$,

$$
M_{i+1}=\bigcup\left\{g\left(M_{i}\right) \cup g^{-1}\left(M_{i}\right): g \in G \text { and } g(L) \subset M_{i}\right\} .
$$

Since $e \in G$ and $L \subset M_{0}$, we have $M_{0} \subset M_{1}$ and, in general, $M_{i} \subset M_{i+1}$. Thus $\left\{M_{i}\right\}$ is an increasing family of sets, and we shall call its union $N$ the extension of $M$ with respect to $L$ and $G$. We observe that if $g \in G$ and $g(L) \subset N$, then $g(N)=N$. For $g(L)$ is finite and so is contained in some $M_{k}$, whence $g\left(M_{i}\right) \subset M_{i+1}$ and $g^{-1}\left(M_{i}\right) \subset M_{i+1}$ for each $i \geqq k$. Hence, $g(N) \subset N, g^{-1}(N) \subset N$, and $g(N)=N$.

Lemma 4. Suppose $X$ is a Hausdorff space, $L$ has $n$ points, $G$ is n-transitive and has the property that, for any net $\left\{g_{k}\right\}$ in $G$ and any $g \in G, \lim _{k} g_{k}(x)=g(x)$ for all $x \in L$ implies

$$
\lim _{k} g_{k}(x)=g(x), \quad \lim _{k} g_{k}^{-1}(x)=g^{-1}(x), \quad x \in X .
$$

Then $g(L) \subset \bar{N}$ implies $g(\bar{N})=\bar{N}$, where $\bar{N}$ is the closure of $N$.

Proof. If $L=\left\{x^{1}, \cdots, x^{n}\right\}$ and $g(L) \subset \bar{N}$, then we can find a net $\left\{\left(x_{k}^{1}, \cdots, x_{k}^{n}\right)\right\}$ of $n$-tuples in $N$ such that $\lim _{k} x_{k}^{i}=g\left(x^{i}\right)(i=1, \cdots, n)$. The $n$-transitivity of $G$ implies that there are elements $g_{k} \in G$ satisfying $g_{k}\left(x^{i}\right)=x_{k}^{i}$ for each $i$ and $k$. Thus

$$
\lim _{k} g_{k}\left(x^{i}\right)=\lim _{k} x_{k}^{i}=g\left(x^{i}\right), \quad i=1, \cdots, n
$$

implies

$$
\lim _{k} g_{k}(x)=g(x), \quad \lim _{k} g_{k}^{-1}(x)=g^{-1}(x), \quad x \in X .
$$

From the remark preceding the lemma, $g_{k}(L) \subset N$ implies $g_{k}(x)$, $g_{k}^{-1}(x) \in N$ for $x \in N$, whence $g(x), g^{-1}(x) \in \bar{N}$ for $x \in N$. Consequently, $g(N) \subset \bar{N}, g(\bar{N}) \subset \bar{N}, g^{-1}(N) \subset \bar{N}, g^{-1}(\bar{N}) \subset \bar{N}$, and $g(\bar{N})=\bar{N}$.

Lemma 5. Let $X$ be m-dimensional euclidean space $E^{m}, G$ the group $A_{m}$ of affine transformations defined on $E^{m}, L$ consist of $m+1$ points which do not lie on any $(m-1)$-dimensional hyperplane, and $M \supset L$ consist of $m+2$ points. Then $N$ is dense in $E^{m}$.

Proof. We recall that the elements $a$ of $A_{m}$ have the form 
$a(x)=t+T x$, where $t \in E^{m}$, and $T$ is a nonsingular linear transformation of $E^{m}$ onto itself. Moreover, $A_{m}$ is strictly $(m+1)$-transitive on $(m+1)$-tuples which do not lie on any $(m-1)$-dimensional hyperplane. We first consider the case $m=1$. The hypothesis of Lemma 4 is clearly satisfied with $n=2$. Let $L=\left\{x_{1}, x_{2}\right\}$ and $M=\left\{x_{1}, x_{2}, x_{3}\right\}$. Evidently we can arrange the indices so that either (i) $x_{1}<x_{2}, x_{1}<x_{3}$ or (ii) $x_{1}>x_{2}, x_{1}>x_{3}$. We will complete the proof for case (i); case (ii) is handled in exactly the same way. Choose $a_{1} \in A_{1}$ so that $a_{1}\left(x_{1}\right)=x_{1}$ and $a_{1}\left(x_{2}\right)=x_{3}$. Then $a_{1}(L) \subset N$, and the remark preceding Lemma 4 implies that $a_{1}(N)=N$. Indeed, $a_{1}^{k}(N)=N$ for any integer $k$, where $a_{1}^{k}$ is the $k$-th iterate of $a_{1}$. Now $a_{1}$ is order-preserving and has just one fixed point at $x_{1}$, so that $\left\{a_{1}^{k}\left(x_{2}\right):-\infty<k<+\infty\right\}$ has $x_{1}$ and $+\infty$ as limit points. In other words, $N$ contains a sequence which converges to $x_{1}$ from the right and another which converges to $+\infty$. If $\bar{N} \neq E^{1}$, then $E^{1}-\bar{N}$ is the union of disjoint open intervals. Let $I=(\lambda, \mu)$ be one of these, where we allow $\lambda=-\infty$ or $\mu=+\infty$. If $\lambda \neq-\infty$, we can find $a_{2} \in A_{1}$ satisfying $a_{2}\left(x_{1}\right)=\lambda$ and $\lambda<a_{2}\left(x_{2}\right) \in N$, whence $a_{2}$ is order-preserving, $a_{2}(L) \subset \bar{N}, a_{2}(\bar{N})=\bar{N}$, and $a_{2}^{-1}(I) \subset E^{1}-\bar{N}$. But $a_{2}^{-1}(\lambda)$ is the left endpoint of $a_{2}^{-1}(I)$, while $a_{2}^{-1}(\lambda)=x_{1}$ has a sequence in $\bar{N}$ converging to it from the right, so that part of this sequence must lie in $a_{2}^{-1}(I)$, which is impossible. If $\lambda=-\infty$, then $\mu \leqq x_{1}$, and we choose $a_{3} \in A_{1}$ so that $a_{3}\left(x_{2}\right)=x_{2}, x_{1}<a_{3}\left(x_{1}\right) \in N$, and $a_{3}\left(x_{1}\right)<x_{2}$. Thus $a_{3}$ is order-preserving, $a_{3}(L) \subset N, a_{3}(N)=N$, and $a_{3}(I) \subset E^{1}-\bar{N}$. But $a_{3}(\mu)>\mu$, and $a_{3}(\mu)$ is the right endpoint of $a_{3}(I)$, whence $\mu \in a_{3}(I)$, which is impossible. Therefore, $\bar{N}=E^{1}$.

We now proceed by induction on $m$. Suppose the lemma has been proved in all dimensions less than a certain $m$,

$$
L=\left\{x_{1}, \cdots, x_{m+1}\right\} \subset\left\{x_{0}, x_{1}, \cdots, x_{m+1}\right\}=M \subset E^{m},
$$

and $L$ does not lie on any $(m-1)$-dimensional hyperplane. We can arrange the indices in $L$ so that either (i) $x_{0}$ lies on the $(m-1)$ dimensional hyperplane $X$ determined by $x_{2}, \cdots, x_{m+1}$, or (ii) $x_{0}$ and $x_{1}$ lie on the same side of $X$. To see this, we set up a coordinate system in $E^{m}$ in which the points of $L$ are the origin and unit points on the coordinate axes. If each point of $L$ lay on the side opposite $x_{0}$ of the $(m-1)$-dimensional hyperplane through the remaining points of $L$, then all the coordinates of $x_{0}$ would be negative, while $x_{0}$ lay on the side opposite the origin of the hyperplane through the unit points, which is impossible. In case (ii), choose $a_{0} \in A_{m}$ so that $a_{0}\left(x_{1}\right)=x_{0}$ and $a_{0}\left(x_{i}\right)=x_{i}(i=2, \cdots, m+1)$. We will show that $x_{1}, a_{0}\left(x_{1}\right)$, and $a_{0}^{2}\left(x_{1}\right)$ are collinear. Since $K\left(a_{0}\right)=X$, we can refer $a_{0}(x)=t_{0}+T_{0} x$ to a coordinate system in $E^{m}$ relative to which $x_{1}=(0, \cdots, 0,1), X$ is the set of points with last coordinate $0, t_{0}=(0, \cdots, 0)$, and $T_{0}$ has 
the form

$$
T_{0}=\left(\begin{array}{ccccc}
1 & 0 & 0 & \cdots & \alpha_{1} \\
0 & 1 & 0 & \cdots & \alpha_{2} \\
0 & 0 & 1 & \cdots & \alpha_{3} \\
\cdots & \cdots & \cdots \\
0 & 0 & 0 & \cdots & \alpha_{m}
\end{array}\right),
$$

Thus we have

$$
\begin{aligned}
& a_{0}\left(x_{1}\right)=\left(\alpha_{1}, \cdots, \alpha_{m-1}, \alpha_{m}\right), \\
& a_{0}^{2}\left(x_{1}\right)=\left(\alpha_{1}\left(1+\alpha_{m}\right), \cdots, \alpha_{m-1}\left(1+\alpha_{m}\right), \alpha_{m}^{2}\right) \text {, } \\
& a_{0}\left(x_{1}\right)-x_{1}=\left(\alpha_{1}, \cdots, \alpha_{m-1}, \alpha_{m}-1\right) \text {, } \\
& a_{0}^{2}\left(x_{1}\right)-a_{0}\left(x_{1}\right)=\left(\alpha_{1} \alpha_{m}, \cdots, \alpha_{m-1} \alpha_{m},\left(\alpha_{m}-1\right) \alpha_{m}\right) \\
& =\alpha_{m}\left(a_{0}\left(x_{1}\right)-x_{1}\right) \text {, }
\end{aligned}
$$

whence $x_{1}, a_{0}\left(x_{1}\right)=x_{0}$, and $a_{0}^{2}\left(x_{1}\right)=a_{0}\left(x_{0}\right)=y_{0}$ are collinear, and $y_{0} \neq x_{0}, x_{1}$. We will show next that there is a subset $L^{\prime}$ of $M$ which contains $x_{0}, x_{1}$, and $m-1$ of the remaining $m$ points of $L$, but which does not lie on any $(m-1)$-dimensional hyperplane. If $L^{\prime}=\left\{x_{0}, x_{1}, \cdots, x_{m}\right\}$ will not work, then let $k$ be the least integer such that $2 \leqq k \leqq m$ and $\left\{x_{0}, x_{1}, \cdots, x_{k}\right\}$ lies on some $(k-1)$-dimensional hyperplane, and set $L^{\prime}=M-\left\{x_{k}\right\}$. Now if $L^{\prime}$ lay on an $(m-1)$-dimensional hyperplane $X_{m-1}$, then the unique $(k-1)$-dimensional hyperplane through $\left\{x_{0}, x_{1}, \cdots, x_{k-1}\right\}$ must contain $x_{k}$ and lie in $X_{m-1}$, so that $M \subset X_{m-1}$, which is impossible. Hence, $L^{\prime}=M-\left\{x_{k}\right\}$ satisfies our condition. Let $x_{j}$ be a fixed element of $L^{\prime}-\left\{x_{0}, x_{1}\right\}, Y$ be the $(m-1)$-dimensional hyperplane through $L^{\prime \prime}=L^{\prime}-\left\{x_{j}\right\}, M^{\prime \prime}=L^{\prime \prime} \cup\left\{y_{0}\right\}$, and $a_{1} \in A_{m}$ map $L$ onto $L^{\prime}$. Since $\left\{y_{0}, x_{0}, x_{1}\right\}$ is collinear, and $x_{0}, x_{1} \in L^{\prime \prime}$, we have $M^{\prime \prime} \subset Y$. Now $L^{\prime \prime}$ contains $m$ points, $M^{\prime \prime}$ contains $m+1$ points, and the group $B$ of elements in $A_{m}$ which fix $x_{j}$ and map $Y$ onto itself acts on $Y$ exactly like $A_{m-1}$. By our induction hypothesis, the extension $N^{\prime \prime}$ of $M^{\prime \prime}$ with respect to $L^{\prime \prime}$ and $B$ is dense in $Y$. We will show that $\bigcup M_{i}^{\prime \prime}=N^{\prime \prime} \subset N=\bigcup M_{i}$ by showing inductively that $M_{i}^{\prime \prime} \subset N$. First, $a_{0}(L) \subset M$ implies $y_{0}=a_{0}\left(x_{0}\right) \in a_{0}(M) \subset N$, so that $M_{0}^{\prime \prime}=M^{\prime \prime} \subset N$. Suppose now that $M_{i}^{\prime \prime} \subset N$ for some $i$, and $b\left(L^{\prime \prime}\right) \subset M_{i}^{\prime \prime}$ for some $b \in B$. Then $a_{1}(L)=L^{\prime} \subset M$ implies $a_{1}(N)=N$, and

$$
b a_{1}(L)=b\left(L^{\prime}\right)=\left\{x_{j}\right\} \cup b\left(L^{\prime \prime}\right) \subset\left\{x_{j}\right\} \cup M_{i}^{\prime \prime} \subset N
$$

implies $b a_{1}(N)=N$. Thus $b(N)=b\left(a_{1}(N)\right)=N, b\left(M_{i}^{\prime \prime}\right) \cup b^{-1}\left(M_{i}^{\prime \prime}\right) \subset N$, and $M_{i+1}^{\prime \prime} \subset N$, so that $N^{\prime \prime} \subset N$. Suppose $\left\{y_{1}, \cdots, y_{m-1}\right\}$ is a subset of $N^{\prime \prime}$ which does not lie in any $(m-3)$-dimensional hyperplane. Since $L^{\prime \prime}$ does not lie on any $(m-2)$-dimensional hyperplane, we can find 
an $x_{i} \in L^{\prime \prime}$ such that $\left\{x_{i}, y_{1}, \cdots, y_{m-1}\right\}$ does not lie on any $(m-2)$ dimensional hyperplane. Then $\left\{x_{i}, x_{j}, y_{1}, \cdots, y_{m-1}\right\}$ does not lie on any $(m-1)$-dimensional hyperplane, and we can find an $a_{2} \in A_{m}$ which maps $L^{\prime}$ onto $\left\{x_{i}, x_{j}, y_{1}, \cdots, y_{m-1}\right\}$ in such a way that $a_{2}\left(x_{i}\right)=x_{j}$ and $a_{2}\left(x_{j}\right)=x_{i}$. From $a_{2} a_{1}(L)=a_{2}\left(L^{\prime}\right) \subset N$, we infer that $a_{2} a_{1}(N)=N$ and $a_{2}(N)=a_{2}\left(a_{1}(N)\right)=N$, so that $a_{2}\left(N^{\prime \prime}\right) \subset N$. Now $a_{2}\left(N^{\prime \prime}\right)$ is a dense subset of $a_{2}(Y)$, and $a_{2}(Y)$ is an $(m-1)$-dimensional hyperplane through $\left\{x_{j}\right\}$ and $\left\{y_{1}, \cdots, y_{m-1}\right\}$. The union of such hyperplanes as $\left\{y_{1}, \cdots, y_{m-1}\right\}$ ranges over $N^{\prime \prime}$ is clearly dense in $E^{m}$, whence $N$ is dense in $E^{m}$, and our main induction step is complete for case (ii). For case (i), the preceding argument becomes considerably simpler. We set

$$
L^{\prime \prime}=\left\{x_{2}, \cdots, x_{m+1}\right\}, \quad M^{\prime \prime}=\left\{x_{0}, x_{2}, \cdots, x_{m+1}\right\},
$$

and let $B$ be the set of elements in $A_{m}$ which fix $x_{1}$ and map $X$ onto itself. Then $N^{\prime \prime} \subset N$, and $N^{\prime \prime}$ is dense in $X$. The last part of the argument with $L^{\prime}=L, Y=X$, and $x_{j}=x_{1}$ shows that $N$ is dense in $E^{m}$ in this case as well.

LEMmA 6. The conclusion of Lemma 5 remains valid if, in the hypothesis, we set $m=1$ and replace $A_{1}$ with the group $A_{1}^{+}$of orderpreserving elements in $A_{1}$.

Proof. We observe that all of the elements in $A_{1}$ which appear in the proof of Lemma 5 are order-preserving. The only other lemma used in that proof was Lemma 4 which assumes that $G$ is 2-transitive. Although $A_{1}^{+}$is only 2-transitive relative to $H^{+}\left(E^{1}\right)$, the net $\left\{g_{k}\right\}$ can still be found, if we recall that any pair of points which lies sufficiently close to a positively oriented pair is also positively oriented.

Lemma 7. Let $X$ be a topological space, $L$ consist of $n$ points, $L \subset M, f \in H(X), G$ and $G^{\prime}$ be subgroups of $H(X)$, and $G^{\prime}$ have the property that if $g^{\prime} \in G^{\prime}$ and $K\left(g^{\prime}\right)$ contains $n$ points, then $g^{\prime}=e$. Suppose that, for every $g \in G$, there is a $g^{\prime} \in G^{\prime}$ such that $f g(x)=g^{\prime} f(x)$ for all $x \in M$. Then $f g(x)=g^{\prime} f(x)$ for all $x$ in the extension $N$ of $M$ with respect to $L$ and $G$.

Proof. We will prove the result inductively for the sets $M=$ $M_{0}, M_{1}, M_{2}, \cdots$. Suppose that, for every $g \in G$, there is a $g^{\prime} \in G^{\prime}$ such that $f g(x)=g^{\prime} f(x)$ for all $x \in M_{i}$, and $g_{1}(L) \subset M_{i}$, where $g_{1} \in G$. If $y \in L$, then $g_{1}(y) \in M_{i}$ and

$$
f g\left(g_{1}(y)\right)=g^{\prime} f\left(g_{1}(y)\right),
$$
$y \in L$.

We know that there are elements $g_{1}^{\prime}, g_{2}^{\prime} \in G^{\prime}$ satisfying 


$$
f g_{1}(y)=g_{1}^{\prime} f(y), \quad f g g_{1}(y)=g_{2}^{\prime} f(y),
$$

Combining (1) and (2) and recalling that $L \subset M_{i}$, we obtain

$$
g_{2}^{\prime} f(y)=f g g_{1}(y)=g^{\prime} f g_{1}(y)=g^{\prime} g_{1}^{\prime} f(y),
$$

$y \in L$.

Thus $f(y) \in K\left(g_{2}^{\prime-1} g^{\prime} g_{1}^{\prime}\right), f(L) \subset K\left(g_{2}^{\prime-1} g^{\prime} g_{1}^{\prime}\right)$, and $f(L)$ contains $n$ points, so that $g_{2}^{\prime-1} g^{\prime} g_{1}^{\prime}=e$ and $g_{2}^{\prime}=g^{\prime} g_{1}^{\prime}$. From (2) we have

$$
f g g_{1}(y)=g_{2}^{\prime} f(y)=g^{\prime} g_{1}^{\prime} f(y)=g^{\prime} f g_{1}(y), \quad y \in M_{i},
$$

that is, $f g(x)=g^{\prime} f(x)$ for all $x \in g_{1}\left(M_{i}\right)$. To see that $f g(x)=g^{\prime} f(x)$ for all $x \in g_{1}^{-1}\left(M_{i}\right)$, we observe that $L \subset M_{i}$ implies

$$
f g g_{1}^{-1}(y)=g^{\prime} f g_{1}^{-1}(y),
$$

We can also find elements $g_{3}^{\prime}, g_{4}^{\prime} \in G^{\prime}$ satisfying

$$
f g_{1}^{-1}(y)=g_{3}^{\prime} f(y), \quad f g g_{1}^{-1}(y)=g_{4}^{\prime} f(y),
$$

$y \in M_{i}$.

From (3), (4), and $g_{1}(L) \subset M_{i}$ we obtain

$$
g_{4}^{\prime} f(y)=f g g_{1}^{-1}(y)=g^{\prime} f g_{1}^{-1}(y)=g^{\prime} g_{3}^{\prime} f(y),
$$

Thus $f g_{1}(L) \subset K\left(g_{4}^{\prime-1} g^{\prime} g_{3}^{\prime}\right)$ and $g_{4}^{\prime}=g^{\prime} g_{3}^{\prime}$. Finally, from (4) we have

$$
f g g_{1}^{-1}(y)=g_{4}^{\prime} f(y)=g^{\prime} g_{3}^{\prime} f(y)=g^{\prime} f g_{1}^{-1}(y), \quad y \in M_{i},
$$

in other words, $f g(x)=g^{\prime} f(x)$ for all $x \in g_{1}^{-1}\left(M_{i}\right)$. Therefore, $f g(x)=$ $g^{\prime} f(x)$ for all $x \in M_{i+1}$, and the induction step is complete.

LEMma 8. With the same hypotheses as in Lemma 7, suppose $G=G^{\prime}$ and $f(x)=x$ for all $x \in M$. Then $f(x)=x$ for all $x \in N$.

Proof. Again we proceed by induction on the sets $M_{i}$. Suppose $f(x)=x$ for all $x \in M_{i}$, and $g_{1}(L) \subset M_{i}$, where $g_{1} \in G$. Then we can find $g_{1}^{\prime} \in G$ such that

$$
f g_{1}(x)=g_{1}^{\prime} f(x)=g_{1}^{\prime}(x), \quad x \in M_{i} .
$$

Since $L, g_{1}(L) \subset M_{i}$, we have

$$
g_{1}(y)=f g_{1}(y)=g_{1}^{\prime}(y),
$$
$y \in L$,

whence $L \subset K\left(g_{1}^{-1} g_{1}^{\prime}\right)$ and $g_{1}=g_{1}^{\prime}$. Thus $f g_{1}(x)=g_{1}(x)$ for all $x \in M_{i}$, that is, $f(z)=z$ for all $z \in g_{1}\left(M_{i}\right)$. Similarly, there is a $g_{2}^{\prime} \in G$ satisfying

$$
\begin{array}{cr}
f g_{1}^{-1}(x)=g_{2}^{\prime} f(x)=g_{2}^{\prime}(x), & x \in M_{i}, \\
g_{1}^{-1}(y)=f g_{1}^{-1}(y)=g_{2}^{\prime}(y), & y \in g_{1}(L),
\end{array}
$$


so that $g_{1}^{-1}=g_{2}^{\prime}$ and $f g_{1}^{-1}(x)=g_{1}^{-1}(x)$ for all $x \in M_{i}$. Therefore, $f(z)=z$ for all $z \in M_{i+1}$, and the induction step is complete.

THeOREM 1. Suppose $X=E^{1}, L$ consists of two points, $M$ of three points, $f \in H^{+}\left(E^{1}\right)$, and, for every $a \in A_{1}^{+}$, there is an $a^{\prime} \in A_{1}^{+}$ such that $f a(x)=a^{\prime} f(x)$ for all $x \in M$. Then $f \in A_{1}^{+}$.

Proof. The hypotheses of Lemma 7 are evidently satisfied when $n=2$ and $G=G^{\prime}=A_{1}^{+}$, whence $f a(x)=a^{\prime} f(x)$ for all $x \in N$. By Lemma $6, N$ is dense in $E^{1}$, and the continuity of $a, a^{\prime}$, and $f$ implies that $f a=a^{\prime} f$, that is, $f A_{1}^{+} f^{-1} \subset A_{1}^{+}$. If we choose $a_{1} \in A_{1}^{+}$so that $a_{1}(0)=f(0), a_{1}(1)=f(1)$, and set $f_{1}=a_{1}^{-1} f$, then $0,1 \in K\left(f_{1}\right)$ and $f_{1} A_{1}^{+} f_{1}^{-1} \subset A_{1}^{+}$. In particular, if we define $a_{2}(x)=1+x$ for $x \in E^{1}$, then $a_{3}=f_{1} a_{2} f_{1}^{-1} \in A_{1}^{+}$. Now $K\left(a_{3}\right)=f_{1}\left(K\left(a_{2}\right)\right)=f_{1}(\varnothing)=\varnothing$, so that $a_{3}$ is also a translation, and $a_{3}(0)=1$ implies $a_{3}=a_{2}$. Thus $2=a_{3}(1)=$ $f_{1} a_{2} f_{1}^{-1}(1)=f_{1}(2)$, and $0,1,2 \in K\left(f_{1}\right)$. Setting $M=\{0,1,2\}$ in Lemmas 6 and 8 , we conclude that $f_{1}=e$ and $f=a_{1} \in A_{1}^{+}$.

4. 3-transitive groups containing $A_{m}$ and $P_{m}$. We are now ready to investigate the transitivity of groups of homeomorphisms of euclidean $m$-space $E^{m}$ or real projective $m$-space $\Pi^{m}$ which contain the affine group $A_{m}$ or the projective group $P_{m}$, respectively, as a proper subgroup. The groups which we will consider are all obtained by adjoining some homeomorphism to $A_{m}$ or $P_{m}$ and generating the smallest group containing them. Any larger group will obviously have at least as high a degree of transitivity. In the case $m=1$, we will obtain slightly sharper results by adjoining an element of $H^{+}\left(E^{1}\right)$ or $H^{+}\left(\Pi^{1}\right)$ to $A_{1}^{+}$or $P_{1}^{+}$, respectively, and considering transitivity relative to $H^{+}\left(E^{1}\right)$ or $H^{+}\left(\Pi^{1}\right)$. Then if an orientation-reversing element of $A_{1}$ or $P_{1}$ is added, the resulting group will clearly have the same degree of transitivity relative to $H\left(E^{1}\right)$ or $H\left(\Pi^{1}\right)$, respectively.

THEOREM 2. If $f \in H^{+}\left(E^{1}\right)-A_{1}$, then the group $G$ generated by $f$ and $A_{1}^{+}$is 3-transitive relative to $H^{+}\left(E^{1}\right)$.

Proof. Given any three points $x_{1}<x_{2}<x_{3}$ in $E^{1}$, let $L=\left\{x_{1}, x_{2}\right\}$ and $M=\left\{x_{1}, x_{2}, x_{3}\right\}$. For each $a \in A_{1}^{+}$, we can find $a^{\prime} \in A_{1}^{+}$satisfying $a^{\prime}\left(f\left(x_{i}\right)\right)=f a\left(x_{i}\right) \quad(i=1,2)$. If $a(x)=\alpha+\beta x$ and $a^{\prime}(x)=\alpha^{\prime}+\beta^{\prime} x$, then $\alpha^{\prime}$ and $\beta^{\prime}$ must satisfy the equations

$$
\begin{aligned}
& \alpha^{\prime}+\beta^{\prime} f\left(x_{1}\right)=f\left(\alpha+\beta x_{1}\right), \\
& \alpha^{\prime}+\beta^{\prime} f\left(x_{2}\right)=f\left(\alpha+\beta x_{2}\right),
\end{aligned}
$$

so that $\alpha^{\prime}$ and $\beta^{\prime}$ are continuous functions of $\alpha$ and $\beta$. We can identify 
$A_{1}^{+}$with the set of pairs $(\alpha, \beta)$ of real numbers, where $\beta>0$. If we give $A_{1}^{+}$the euclidean topology of a half-plane and hold $x \in E^{1}$ fixed, then the mapping $a \rightarrow a(x)$ or $(\alpha, \beta) \rightarrow \alpha+\beta x$ from $A_{1}^{+}$into $E^{1}$ is evidently continuous. Since $f$ and $f^{-1}$ are continuous, so also is the mapping $a \rightarrow \varphi(a)=f^{-1} a^{\prime-1} f a\left(x_{3}\right)$ from $A_{1}^{+}$into $E^{1}$. From Theorem 1, we know that there is at least one $a_{0} \in A_{1}^{+}$such that $a_{0}^{\prime} f\left(x_{3}\right) \neq f a_{0}\left(x_{3}\right)$, for otherwise $f \in A_{1}^{+}$, contrary to our hypothesis. Thus $\varphi\left(a_{0}\right) \neq x_{3}$ while $\varphi(e)=x_{3}$. From the connectedness of $A_{1}^{+}$we infer that $\varphi\left(A_{1}^{+}\right)$ is a nondegenerate interval and so contains an open set. Moreover, $f^{-1} a^{\prime-1} f a \in G$ and $x_{1}, x_{2} \in K\left(f^{-1} a^{\prime-1} f a\right)$. By Lemma $3, G$ is 3-transitive relative to $H^{+}\left(E^{1}\right)$.

THEOREM 3. If $m \geqq 2$ and $f \in H\left(E^{m}\right)-A_{m}$, then the group $G$ generated by $f$ and $A_{m}$ is 3-transitive.

Proof. We know that $A_{m}$ maps any noncollinear triple onto any other noncollinear triple. If we can show that $G$ maps every collinear triple onto some noncollinear triple, then we will have established that $G$ is 3-transitive. Let $M$ be a collinear triple, $L \subset M$ consist of two points, $X$ be the line through $M$, and suppose that, for every $a \in A_{m}$, $f a(M)$ is a collinear triple. The group $B$ of all those elements in $A_{m}$ which map $X$ onto itself behaves exactly like $A_{1}$ on $X$. By Lemma 5 , the extension $N$ of $M$ with respect to $L$ and $B$ is dense in $X$. We will show by induction on the sets $M_{i}$ that, for every $a \in A_{m}$, $f a(N)$ is a collinear set. Suppose $f a\left(M_{i}\right)$ is a collinear set for each $a \in A_{m}$, and $b(L) \subset M_{i}$ for some $b \in B$. Then $f a\left(b\left(M_{i}\right)\right)=f a b\left(M_{i}\right)$ and $f a\left(b^{-1}\left(M_{i}\right)\right)=f a b^{-1}\left(M_{i}\right)$ are each collinear, and

$$
\begin{aligned}
& f a\left(M_{i}\right) \cap f a\left(b\left(M_{i}\right)\right) \supset f a(b(L)), \\
& f a\left(M_{i}\right) \cap f a\left(b^{-1}\left(M_{i}\right)\right) \supset f a(L) .
\end{aligned}
$$

Since $f a(b(L))$ and $f a(L)$ each contain two points, the sets $f a\left(M_{i}\right)$, $f a\left(b\left(M_{i}\right)\right)$, and $f a\left(b^{-1}\left(M_{i}\right)\right)$ all lie on the same line, so that $f a\left(M_{i+1}\right)$ is collinear, and the induction step is complete. From $\bar{N}=X$ we infer that $f a(X)$ is collinear for each $a \in A_{m}$. If $Y$ is any line in $E^{m}$, then we can choose $a_{0} \in A_{m}$ such that $a_{0}(X)=Y$, whence $f(Y)=f a_{0}(X)$ is also collinear. Since $Y$ is closed, connected, and separated by each of its points, the same must also be true of $f(Y)$ so that $f(Y)$ is a line. Let $Y_{1}, Y_{2}$ be parallel lines and $Z$ a line which meets them both. Then $Y_{1} \cap Y_{2}=\varnothing$, and any line which meets $Z$ and $Y_{1}$ in distinct points mush also meet $Y_{2}$. Since $f$ preserves these incidence relations, we conclude that $f\left(Y_{1}\right)$ and $f\left(Y_{2}\right)$ are parallel. Let $L^{\prime}$ consist of the origin and the $m$ unit points in a coordinate system for $E^{m}$, and let $M^{\prime}$ be the set of $2^{m}$ vertices of the unit cube determined by $L^{\prime}$. 
Then $f a\left(M^{\prime}\right)$ is the set of vertices of a parallelotope for each $a \in A_{m}$, and we can find $a^{\prime} \in A_{m}$ satisfying $f a(x)=a^{\prime} f(x)$ for all $x \in M^{\prime}$. If we select $a_{1} \in A_{m}$ so that $a_{1}(x)=f(x)$ for all $x \in M^{\prime}$ and set $f_{1}=a_{1}^{-1} f$, then $M^{\prime} \subset K\left(f_{1}\right)$ and

$$
f_{1} a(x)=a_{1}^{-1} f a(x)=a_{1}^{-1} a^{\prime} f(x)=a_{1}^{-1} a^{\prime} a_{1} f_{1}(x), \quad x \in M^{\prime} .
$$

We infer from Lemmas 5 and 8 that $f_{1}=e$ and $f=a_{1}$, which contradicts the hypothesis of our theorem. Hence, $f a(M)$ is not collinear for some $a \in A_{m}$.

The conclusion of Theorem 3 seems especially weak in view of the fact that $A_{m}$ itself is $(m+1)$-transitive on subsets which do not lie on any $(m-1)$-dimensional hyperplane. The difficulty in extending our method to higher transitivity comes from (5). If we knew, for example, that $f a(b(L))$ and $f a(L)$ each contained three points, it would not follow that these triples were noncollinear, and we could not conclude that $f a\left(M_{i}\right), f a\left(b\left(M_{i}\right)\right)$, and $f a\left(b^{-1}\left(M_{i}\right)\right)$ were coplanar.

Lemma 9. Suppose the group $F$ generated by $A_{1}^{+}$and $f \in H^{+}\left(E^{1}\right)$ is n-transitive relative to $H^{+}\left(E^{1}\right)$. If we extend $f$ to an element $\bar{f}$ of $H^{+}\left(\Pi^{1}\right)$ by making $\bar{f}$ fix the point at infinity, then the group $G$ generated by $P_{1}^{+}$and $\bar{f}$ is $(n+1)$-transitive relative to $H^{+}\left(\Pi^{1}\right)$.

Proof. An element $p \in P_{1}^{+}=P_{1} \cap H^{+}\left(\Pi^{1}\right)$ has the form $p(x)=$ $(\alpha x+\beta) /(\gamma x+\delta)$, where $\alpha \delta^{\prime}-\beta \gamma>0$. We can identify $A_{1}^{+}$with the subgroup of $P_{1}^{+}$which leaves fixed the point $\infty$ at infinity. Suppose that $\left\{x_{1}, \cdots, x_{n+1}\right\}$ and $\left\{y_{1}, \cdots, y_{n+1}\right\}$ are given such that, as $i$ increases from 1 to $n+1, x_{i}$ and $y_{i}$ each move in the positive sense of orientation. Choose $p_{0}, p_{1} \in P_{1}^{+}$so that $p_{0}\left(x_{1}\right)=\infty$ and $p_{1}\left(y_{1}\right)=\infty$. Then $\left\{p_{0}\left(x_{2}\right), \cdots, p_{0}\left(x_{n+1}\right)\right\},\left\{p_{1}\left(y_{2}\right), \cdots, p_{1}\left(y_{n+1}\right)\right\} \subset \Pi^{1}-\{\infty\}$, and the points in each set increase with $i$. Thus we can find $g_{0} \in F$ satisfying $g_{0}\left(p_{0}\left(x_{i}\right)\right)=p_{1}\left(y_{i}\right)(i=2, \cdots, n+1)$, and $g_{1}=p_{1}^{-1} \bar{g}_{0} p_{0} \in G$ must satisfy $g_{1}\left(x_{i}\right)=y_{i}(i=1, \cdots, n+1)$.

THEOREM 4. If $f \in H^{+}\left(\Pi^{1}\right)-P_{1}^{+}$, then the group $G$ generated by $f$ and $P_{1}^{+}$is 4-transitive relative to $H^{+}\left(\Pi^{1}\right)$.

Proof. Let $f(\infty)=x_{0}$, and choose $p_{0} \in P_{1}^{+}$so that $p_{0}\left(x_{0}\right)=\infty$. Then $p_{0} f(\infty)=\infty$, and the restriction $f_{0}$ of $p_{0} f$ to $\Pi^{1}-\{\infty\}=E^{1}$ belongs to $H^{+}\left(E^{1}\right)$. Theorem 2 says that the group $F$ generated by $f_{0}$ and the set $A_{1}^{+}$of those elements of $P_{1}^{+}$which fix $\infty$ is 3-transitive relative to $H^{+}\left(E^{1}\right)$, and Lemma 9 gives the desired result.

THEOREM 5. If $m \geqq 2$ and $f \in H\left(\Pi^{m}\right)-P_{m}$, then the group $G$ generated by $f$ and $P_{m}$ is 3-transitive. 
Proof. Since $P_{m}$ maps any noncollinear triple onto any other noncollinear triple, our result will be proved if we can show that, for any collinear triple $M$, there is a $p \in P_{m}$ such that $f p(M)$ is noncollinear. Suppose that, for some collinear triple $M=\left\{x_{1}, x_{2}, x_{3}\right\}$ and every $p \in P_{m}$, $f p(M)$ is collinear. Let $X$ be a projective line in $\Pi^{m}, p_{0} \in P_{m}$ map $M$ into $X$, and $Q$ be the subgroup of $P_{m}$ which maps $X$ onto itself. We know that $Q$ acts like $P_{1}$ on $X$ and is, therefore, 3-transitive without regard to orientation. Let $x \in X-\left\{p_{0}\left(x_{1}\right), p_{0}\left(x_{2}\right)\right\}$ be arbitrary, and choose $q \in Q$ so that $\left\{p_{0}\left(x_{1}\right), p_{0}\left(x_{2}\right)\right\} \subset K(q)$ and $q\left(p_{0}\left(x_{3}\right)\right)=x$. Then $f q\left(p_{0}(M)\right)$ and $f\left(p_{0}(M)\right)$ are each collinear and have two points in common, so that $f(x)$ lies on the projective line $Y$ through $f\left(p_{0}(M)\right)$, and $f(X) \subset Y$. Since $f$ is a homeomorphism, and $X, Y$ are topological circles, we must have $f(X)=Y$. If $Z$ denotes the $(m-1)$-dimensional projective hyperplane at infinity, then any projective line which meets $Z$ in two points must lie in $Z$. Moreover, $f(Z)$ must have the same property, for $f$ preserves incidence relations. Hence, $f(Z)$ is a projective hyperplane, and $f(Z)$ has dimension $m-1$. If we choose $p_{1} \in P_{m}$ so that $p_{1}(Z)=f(Z)$ and set $f_{1}=p_{1}^{-1} f$, then $f_{1}(Z)=Z$, and the restriction $f_{1}^{*}$ of $f_{1}$ to $\Pi^{m}-Z=E^{m}$ maps lines onto lines. Following the argument in the proof of Theorem 3, we infer that $f_{1}^{*}$ is affine, $f_{1} \in P_{m}$, and $f \in P_{m}$, which contradicts the hypothesis of our theorem. Therefore, $f p(M)$ is noncollinear for some $p \in P_{m}$.

5. $\omega$-transitive groups. So far, we have not exhibited any $f$ such that the group generated by $f$ and $A_{m}$ is $\omega$-transitive. This we will now do. As before, the results for the case $m=1$ are much stronger than those for $m>1$, and this seems to be due to the fact that a nondegenerate connected subset of $E^{1}$ has a nonempty interior. The conditions which we shall impose on $f$ all have to do with its fixed point set and require, at the very least, that this should have a nonempty interior.

THEOREM 6. Suppose $f \in H^{+}\left(E^{1}\right), f \neq e$, and $K(f)$ contains a halfline. Then the group $G$ generated by $f$ and the set $B$ of all translations in $A_{1}^{+}$is $\omega$-transitive relative to $H^{+}\left(E^{1}\right)$.

Proof. Let $x_{1}<\cdots<x_{n+1}$ be arbitrary points of $E^{1}$, and suppose $\left(-\infty, x_{0}\right]$ is a connected component of $K(f)$. The case $\left[x_{0},+\infty\right) \subset K(f)$ is handled in the same way. Choose $b_{0} \in B$ so that $b_{0}\left(x_{0}\right)=x_{n+1}$. If we set $f_{0}=b_{0} f b_{0}^{-1}$, then $K\left(f_{0}\right)=b_{0}(K(f))$ has $\left(-\infty, x_{n+1}\right]$ as a connected component. The elements of $B$ have the form $b(x)=\beta+x$, and if we give to $B$ the topology induced by the euclidean topology for $\beta$, then the mapping $\varphi(b)=b f_{0} b^{-1}\left(x_{n+1}\right)$ from $B$ into $E^{1}$ becomes continuous. Now $\varphi(e)=f_{0}\left(x_{n+1}\right)=x_{n+1}$, and we can find a connected neighborhood 
$U \subset B$ of $e$ so that $b \in U$ implies $b\left(x_{n+1}\right) \in\left(x_{n},+\infty\right)$. Since $x_{n+1}$ is a boundary point of $K\left(f_{0}\right)$, we can find $b \in U$ with the property that $b^{-1}\left(x_{n+1}\right) \in E^{1}-K\left(f_{0}\right)$, whence $\varphi(b) \neq x_{n+1}$. From the connectedness of $U$ we infer that $\varphi(U)$ is a nondegenerate interval which must have a nonempty interior. If we set $G_{0}=\left\{g \in G:\left\{x_{1}, \cdots, x_{n}\right\} \subset K(g)\right\}$, then $b \in U$ implies

$$
K\left(b f_{0} b^{-1}\right) \supset b\left(\left(-\infty, x_{n+1}\right]\right)=\left(-\infty, b\left(x_{n+1}\right)\right] \supset\left(-\infty, x_{n}\right],
$$

so that $b f_{0} b^{-1} \in G_{0}$ and $\varphi(U) \subset G_{0}\left(x_{n+1}\right)$. Lemma 3 tells us that if we know $G$ to be $n$-transitive relative to $H^{+}\left(E^{1}\right)$, then $G$ is $(n+1)$-transitive. Since $G$ is clearly 0 -transitive, a simple induction argument shows that $G$ is $\omega$-transitive.

Clearly the group $G_{2}$ generated by $f$ and any conjugate $h B h^{-1}$ of $B$, where $h \in H\left(E^{1}\right)$, is also $\omega$-transitive relative to $H^{+}\left(E^{1}\right)$. For the fixed point set of $f_{1}=h^{-1} f h$ is homeomorphic to that of $f$, so that the group $G_{1}$ generated by $f_{1}$ and $B$ is $\omega$-transitive by Theorem 6 , and $G_{2}=h G_{1} h^{-1}$. Similar remarks apply to the other theorems in this section. We also observe that some groups generated by $f \in H^{+}\left(E^{1}\right)-A_{1}^{+}$ and $B$ are not even 2-transitive. Choose $b_{0} \in B$ and $f \in H^{+}\left(E^{1}\right)-A_{1}^{+}$ so that $b_{0}(x)=\beta_{0}+x$, where $\beta_{0} \neq 0$, and $f$ has period $\beta_{0}$ in the sense that $f\left(\beta_{0}+x\right)=\beta_{0}+f(x)$, or $b_{0} f b_{0}^{-1}=f$. Now $f$ and each element of $B$ commutes with $b_{0}$, so every element of the group $G$ generated by $f$ and $B$ commutes with $b_{0}$. If any such element maps $x$ into $y$, then it maps $x+\beta_{0}$ into $y+\beta_{0}$, and $G$ is not 2-transitive.

THEOREM 7. Suppose $\left\{f_{1}, f_{2}, \cdots\right\} \subset H^{+}\left(E^{1}\right)$, and, for every compact subset $C$ of $E^{1}$, there is an $f_{m}$ satisfying $E^{1} \neq K\left(f_{m}\right) \supset C$. Then the group $G$ generated by $\left\{f_{1}, f_{2}, \cdots\right\}$ and $B$ is $\omega$-transitive relative to $H^{+}\left(E^{1}\right)$.

Proof. Let $x_{1}<\cdots<x_{n+1}$ be arbitrary points in $E^{1}$, and $f_{m}$ have the property that $E^{1} \neq K\left(f_{m}\right) \supset\left[x_{1}-1, x_{n+1}\right]$. If $K\left(f_{m}\right)$ contains a half-line, then our result follows from Theorem 6 . We will assume, therefore, that the connected component $\left[y_{0}, y_{1}\right]$ of $K\left(f_{m}\right)$ which contains $\left[x_{1}-1, x_{n+1}\right]$ is bounded. Choose $b_{0} \in B$ so that $b_{0}\left(y_{1}\right)=x_{n+1}$, set $g_{0}=$ $b_{0} f_{m} b_{0}^{-1}$, and let $\varphi(b)=b g_{0} b^{-1}\left(x_{n+1}\right)$ for each $b \in B$. Then $K\left(g_{0}\right)$ has $\left[y_{2}, x_{n+1}\right]$ as a connected component, where $y_{2}=b_{0}\left(y_{0}\right) \leqq x_{1}-1$. As in the proof of Theorem $6, \varphi$ is continuous, $\varphi(e)=x_{n+1}$, and we can find a connected neighborhood $U \subset B$ of $e$ such that $b \in U$ implies $b\left(x_{n+1}\right) \in\left(x_{n},+\infty\right)$ and $b\left(y_{2}\right) \in\left(-\infty, x_{1}\right)$. Again there is a $b \in U$ such that $\varphi(b) \neq x_{n+1}$, and if we define $G_{0}$ as before, then $b \in U$ implies

$$
K\left(b g_{0} b^{-1}\right) \supset b\left(\left[y_{2}, x_{n+1}\right]\right) \supset\left[x_{1}, x_{n}\right] \text {, }
$$


so that $b g_{0} b^{-1} \in G_{0}$ and $\varphi(U) \subset G_{0}\left(x_{n+1}\right)$. The rest of the proof follows that of Theorem 6 .

THeOREM 8. Suppose $f, g \in H^{+}\left(E^{1}\right), E^{1} \neq K(f)$ has a nonempty interior, and $K(g)=\left\{y_{0}\right\}$. Then the group $G$ generated by $f, g$ and $B$ is $\omega$-transitive relative to $H^{+}\left(E^{1}\right)$.

Proof. Choose $y_{1}, y_{2} \in E^{1}$ and $b_{0} \in B$ so that $\left[y_{1}, y_{2}\right] \subset K(f)$ and $b_{0}\left(y_{0}\right)=y_{1}$. If we set $g_{0}=b_{0} g b_{0}^{-1}$, then $K\left(g_{0}\right)=\left\{y_{1}\right\}$, and if we define $g_{1}=g_{0}$ in case $g_{0}\left(y_{2}\right)>y_{2}$ and $g_{1}=g_{0}^{-1}$ in case $g_{0}^{-1}\left(y_{2}\right)>y_{2}$, then $g_{1}^{m}\left(y_{2}\right) \rightarrow$ $+\infty$ as $m \rightarrow+\infty$. Finally, let $b_{m}(x)=\beta_{m}+x$ and

$$
f_{m}=b_{m}^{-1} g_{1}^{m} f g_{1}^{-m} b_{m} \text {. }
$$

Then

$$
\begin{aligned}
K\left(f_{m}\right) & =b_{m}^{-1} g_{1}^{m}(K(f)) \supset b_{m}^{-1}\left(\left[y_{1}, g_{1}^{m}\left(y_{2}\right)\right]\right) \\
& =\left[-\beta_{m}+y_{1},-\beta_{m}+g_{1}^{m}\left(y_{2}\right)\right] .
\end{aligned}
$$

If we choose $\beta_{m}=g_{1}^{m}\left(y_{2}\right) / 2$, then any compact subset of $E^{1}$ will eventually lie in some $K\left(f_{m}\right)$, and our result follows from Theorem 7 .

CoRollary. With the same hypotheses as in Theorem 8, the group generated by $f$ and $A_{1}^{+-}$is $\omega$-transitive relative to $H^{+}\left(E^{1}\right)$.

Theorem 9. Suppose $\left\{f_{1}, f_{2}, \cdots\right\} \subset H^{+}\left(\Pi^{1}\right)$, and there is a point $y_{0} \in \Pi^{1}$ such that, for every neighborhood $U$ of $y_{0}$, we can find an $f_{m}$ satisfying $\Pi^{1} \neq K\left(f_{m}\right) \supset \Pi^{1}-U$. Then the group $G$ generated by $\left\{f_{1}, f_{2}, \cdots\right\}$ and $Q$ is $\omega$-transitive relative to $H^{+}\left(\Pi^{1}\right)$, where $Q$ is the group of "rotations" $q \in P_{1}^{+}$of the form $q(x)=(\alpha x-\beta) /(\beta x+\alpha)$ with $\alpha, \beta$ real and not both 0 .

Proof. The name "rotation" for an element of $Q$ is suggested by the fact that $Q$ is strictly 1-transitive, so that $e$ is the only one of its elements with fixed points. We can identify $Q$ with the set of ordered pairs $(\alpha, \beta)$, excluding $(0,0)$, but we must also identify $(\alpha, \beta)$ with $(\lambda \alpha, \lambda \beta)$ for each real $\lambda \neq 0$. Thus $Q$ is topologically equivalent to $\Pi^{1}$, that is, a circle. The action of $Q$ on $\Pi^{1}$ is, therefore, the same as that of the group of real numbers modulo $2 \pi$ on itself by means of left translation. We will show, first of all, that the group $G_{1}$ of those elements in $G$ which fix $\infty$ is $\omega$-transitive relative to $H^{+}\left(E^{1}\right)$. Let $x_{1}<\cdots<x_{n+1} \in E^{1} \subset \Pi^{1}$ be arbitrary, $q_{0} \in Q$ map $y_{0}$ into $x_{n+1}+1$, and $f_{m}$ have the property that

$$
\Pi^{1} \neq K\left(f_{m}\right) \supset \Pi^{1}-q_{0}^{-1}\left(\left(x_{n+1}, x_{n+1}+2\right)\right) .
$$


Setting $f=q_{0} f_{m} q_{0}^{-1}$, we have $\Pi^{1} \neq K(f) \supset \Pi^{1}-\left(x_{n+1}, x_{n+1}+2\right)$. Let $y_{1}$ be the right-hand endpoint of the connected component $D$ of $K(f)$ which contains $\Pi^{1}-\left(x_{n+1}, x_{n+1}+2\right)$, where $\Pi^{1}$ is oriented so as to agree with the ordering of $E^{1}$. If we choose $q_{1} \in Q$ so that $q_{1}\left(y_{1}\right)=x_{n+1}$ and set $g_{1}=q_{1} f q_{1}^{-1}$, then $q_{1}(D)$ is a connected component of $K\left(g_{1}\right)$ which contains $\Pi^{1}-\left(x_{n+1}, x_{n+1}+2\right)$. We define $\varphi(q)=q g_{1} q^{-1}\left(x_{n+1}\right)$ for each $q \in Q$, and observe that $\varphi$ is continuous, $\varphi(e)=x_{n+1}$, and there is a connected neighborhood $V \subset Q$ of $e$ such that $q \in V$ implies $q\left(\left(x_{n+1}, x_{n+1}+2\right)\right) \subset\left(x_{n},+\infty\right)$. As before, $\varphi(V)$ has a nonempty interior, and $q \in V$ implies

$$
K\left(q g_{1} q^{-1}\right) \supset q\left(\Pi^{1}-\left(x_{n+1}, x_{n+1}+2\right)\right) \supset \Pi^{1}-\left(x_{n},+\infty\right),
$$

so that $q g_{1} q^{-1} \in G_{1}$. If we set $G_{0}=\left\{g \in G_{1}:\left\{x_{1}, \cdots, x_{n}\right\} \subset K(g)\right\}$, then $G_{0}\left(x_{n+1}\right)$ has a nonempty interior, and Lemma 3 implies that $G_{1}$ is $\omega$-transitive relative to $H^{+}\left(E^{1}\right)$. To show that $G$ is $\omega$-transitive relative to $H^{+}\left(\Pi^{1}\right)$, we can apply the argument in the proof of Lemma 9 with $P_{1}^{+}$replaced by $Q$, for only the 1-transitivity of $P_{1}^{+}$was used in that case.

Theorem 10. Suppose $f, g \in H^{+}\left(\Pi^{1}\right), \Pi^{1} \neq K(f)$ has a nonempty interior, and $K(g)=\left\{y_{0}\right\}$. Then the group $G$ generated by $f, g$ and $Q$ is $\omega$-transitive relative to $H^{+}\left(\Pi^{1}\right)$.

Proof. Choose $y_{1}<y_{2}$ in $E^{1} \subset \Pi^{1}$ and $q_{0}, q_{1} \in Q$ so that $\left[y_{1}, y_{2}\right] \subset K(f)$, $q_{0}\left(y_{0}\right)=\infty$, and $q_{1}\left(y_{1}\right)=\infty$. Then $g_{0}=q_{0} g q_{0}^{-1}$ has only one fixed point at $\infty$, and $f_{0}=q_{1} f q_{1}^{-1}$ leaves fixed the points of $\left[-\infty, y_{3}\right]$, where $y_{3}=q_{1}\left(y_{2}\right)$ and, for the sake of our interval notation, we identify $-\infty$ and $+\infty$ with $\infty$. Now $\left\{g_{0}^{k}\left(y_{3}\right):-\infty<k<+\infty\right\}$ has $+\infty$ as a limit point, and, for every neighborhood $U$ of $\infty$, we can find an integer $k$ satisfying

$$
\Pi^{1}-U \subset\left[-\infty, g_{0}^{k}\left(y_{3}\right)\right] \subset K\left(g_{0}^{k} f_{0} g_{0}^{-k}\right) .
$$

Our result now follows from Theorem 9 .

CoROLlaRY. With the same hypotheses as in Theorem 10, the group generated by $f$ and $P_{1}^{+}$is $\omega$-transitive relative to $H^{+}\left(\Pi_{1}\right)$.

THEOREM 11. Suppose $X$ is a locally compact, locally connected metric space which can not be separated by any finite set,

$$
\left\{f_{1}, f_{2}, \cdots\right\} \subset H(X),
$$

and $y_{0} \in X$ has the property that $\left\{X-K\left(f_{k}\right)\right\}$ is a base for the neighborhoods of $y_{0}$. Let $R \subset H(X)$ be a 1-transitive group of 
isometries of $X$, and $R_{0}=\left\{r \in R: r\left(y_{0}\right)=y_{0}\right\}$. Suppose there is a continuous mapping $\sigma$ from $[0,1]$ into $R$ with the topology of uniform convergence on compact sets such that $\sigma(0) \in R_{0}, \sigma(1) \in R-R_{0}$, and, for each $y \in X, R_{0}(y)$ is the sphere containing $y$ with center at $y_{0}$. Then the group $G$ generated by $\left\{f_{1}, f_{2}, \cdots\right\}$ and $R$ is $\omega$-transitive.

Proof. Let $x_{1}, \cdots, x_{n+1} \in X$ be given, and

$$
G_{0}=\left\{g \in G:\left\{x_{1}, \cdots, x_{n}\right\} \subset K(g)\right\} .
$$

If we can show that $G_{0}\left(x_{n+1}\right)$ has a nonempty interior, then our result will follow by induction from Lemma 1 . Since $G$ is 1-transitive, we may assume that $x_{n+1}=y_{0}$. For let $g_{0} \in G$ map $x_{n+1}$ into $y_{0}$, and

$$
G_{0}^{\prime}=\left\{g^{\prime} \in G:\left\{g_{0}\left(x_{1}\right), \cdots, g_{0}\left(x_{n}\right)\right\} \subset K\left(g^{\prime}\right)\right\} .
$$

Then $g \in G_{0}$ implies $g_{0} g g_{0}^{-1} \in G_{0}^{\prime}$, and $g^{\prime} \in G_{0}^{\prime}$ implies $g_{0}^{-1} g^{\prime} g_{0} \in G_{0}$, whence $g_{0}^{-1} G_{0}^{\prime} g_{0}=G_{0}$. If we know that $G_{0}^{\prime}\left(y_{0}\right)$ has a nonempty interior, then

$$
G_{0}\left(x_{n+1}\right)=g_{0}^{-1} G_{0}^{\prime} g_{0}\left(x_{n+1}\right)=g_{0}^{-1}\left(G_{0}^{\prime}\left(y_{0}\right)\right)
$$

also has a nonempty interior. Hence, we can assume that $x_{n+1}=y_{0}$. If we set $\sigma(t)=r_{t}$ for $t \in[0,1]$, then $\alpha=\rho\left(r_{1}\left(y_{0}\right), y_{0}\right)>0$, where $\rho$ is the metric for $X$. Let $\beta$ be the shortest distance from $y_{0}$ to $\left\{x_{1}, \cdots, x_{n}\right\}, U_{\varepsilon}$ the open ball with center $y_{0}$ and radius $\varepsilon=\min (\alpha, \beta / 2)$, and $f_{k}$ such that $y_{0} \in X-K\left(f_{k}\right) \subset U_{\varepsilon}$. Since $\varepsilon \leqq \alpha$, and $\rho\left(r_{t}\left(y_{0}\right), y_{0}\right)$ is a continuous function of $t$, we can find $\delta \in[0,1]$ satisfying $\rho\left(r_{t}\left(y_{0}\right), y_{0}\right) \leqq \varepsilon$ for $t \in[0, \delta]$ and $\rho\left(r_{\delta}\left(y_{0}\right), y_{0}\right)=\varepsilon$. This also implies that $\rho\left(y_{0}, r_{t}^{-1}\left(y_{0}\right)\right) \leqq \varepsilon$ for $t \in[0, \delta]$. If we set

$$
G_{1}=\left\{s r_{t}^{-1} f_{k} r_{t} s^{-1}: t \in[0, \delta], s \in R_{0}\right\},
$$

then $G_{1} \subset G_{0}$. For

$$
\begin{aligned}
K\left(s r_{t}^{-1} f_{k} r_{t} s^{-1}\right) & =s r_{t}^{-1}\left(K\left(f_{k}\right)\right) \supset X-s r_{t}^{-1}\left(U_{\varepsilon}\right) \supset X-s\left(U_{2 \varepsilon}\right) \\
& =X-U_{2 \varepsilon} \supset\left\{x_{1}, \cdots, x_{n}\right\} .
\end{aligned}
$$

Moreover,

$$
r_{t}^{-1} f_{k} r_{t}\left(y_{0}\right) \in r_{t}^{-1} f_{k}\left(\bar{U}_{\varepsilon}\right) \subset r_{t}^{-1}\left(\bar{U}_{\varepsilon}\right) \subset \bar{U}_{2 \varepsilon},
$$

and if we hold $t$ fixed and let $s$ vary, then

$$
s r_{t}^{-1} f_{k} r_{t} s^{-1}\left(y_{0}\right)=s\left(r_{t}^{-1} f_{k} r_{t}\left(y_{0}\right)\right)
$$

is a sphere with center $y_{0}$ and radius

$$
\theta(t)=\rho\left(y_{0}, r_{t}^{-1} f_{k} r_{t}\left(y_{0}\right)\right), \quad t \in[0, \delta] .
$$

Since $r_{\delta}\left(y_{0}\right)$ lies on the boundary of $\bar{U}_{\varepsilon}$, we have $r_{\delta}^{-1} f_{k} r_{\delta}\left(y_{0}\right)=y_{0}$, and 
since $r_{0}\left(y_{0}\right)=y_{0} \in X-K\left(f_{k}\right)$, we have $r_{0}^{-1} f_{k} r_{0}\left(y_{0}\right) \neq y_{0}$. Thus $\theta(0) \neq 0$, and $\theta(\delta)=0$. Now the local compactness and local connectedness of $X$ implies that the mapping $h \rightarrow h^{-1}$ is continuous, and $(h, x) \rightarrow h(x)$ is jointly continuous in the topology of uniform convergence on compact sets [1], so that $\theta:[0, \delta] \rightarrow E^{1}$ is continuous, and $\theta([0, \delta])$ is a nondegenerate interval. Hence, $G_{1}\left(y_{0}\right)$ contains all spheres with center $y_{0}$ and radius less than some positive number, and $G_{1}\left(y_{0}\right) \subset G_{0}\left(y_{0}\right)$ has a nonempty interior.

CoRollary 1. With the same hypotheses as in Theorem 11, suppose that we have $f, g \in H(X)$ with the property that $\left\{g^{k}(X-K(f)): k \geqq 0\right\}$ is a base for the neighborhoods of $y_{0}$. Then the group generated by $f, g$, and $R$ is $\omega$-transitive.

Proof. We set $f_{k}=g^{k} f g^{-k}$ and apply Theorem 11 .

CoRollary 2. Suppose $X=E^{m}$ ( $\left.m \geqq 2\right), R$ is the group of rigid motions of $E^{m}, y_{0} \in E^{m}$, and $\left\{f_{1}, f_{2}, \cdots\right\}$ is as in the hypothesis of Theorem 11. Then $G$ is $\omega$-transitive.

Proof. For the mapping $\sigma$, we set $r_{t}(x)=t x_{0}+x$, where $x_{0} \neq 0$ is a fixed point of $E^{m}$.

CoRollary 3. Suppose $X=\Pi^{m}$ ( $\left.m \geqq 2\right), R$ is the set of elements in $P_{m}$ which can be represented by $(m+1)$-th order unitary matrices, $y_{0} \in \Pi^{m}$, and $\left\{f_{1}, f_{2}, \cdots\right\}$ is as in the hypothesis of Theorem 11. Then $G$ is $\omega$-transitive.

Proof. If we regard $\Pi^{m}$ as the unit sphere in $E^{m+1}$ with antipodal points identified and the metric induced by $E^{m+1}$, then the elements of $R$ are isometries of $\Pi^{m}$. For the mapping $\sigma$, we choose a oneparameter subgroup of rotations about some axis which does not pass through $y_{0}$.

Lemma 10. Let $X$ be a topological space, $G$ a subgroup of $H(X)$, $\varphi$ a homeomorphism from $E^{1}$ onto a closed subset $Y$ of $X$, and $F=$ $\{g \in G: g(Y)=Y\}$. Suppose $\varphi^{-1} F \varphi$ contains $A_{1}$, and there is a $g_{0} \in G$ with the properties $K\left(g_{0}\right) \supset \varphi([0,1])$ and $g_{0}(Y)-Y \neq \varnothing$. Then for any interval $I=[\alpha, \beta]$ in $E^{1}$ and any $y \in Y-\varphi(I)$, we can find $a$ $g \in G$ such that $K(g) \supset \varphi(I)$ and $g(y) \in X-Y$.

Proof. Let $G_{0}=\{g \in G: \varphi(I) \subset K(g)\}$ and $Y_{0}=\left\{y \in Y: G_{0}(y)-Y \neq \varnothing\right\}$. Clearly $Y_{0}$ is open in $Y$. If $a \in A_{1}$ and $a(I) \supset I$, then we will show that $a \varphi^{-1}\left(Y_{0}\right) \subset \varphi^{-1}\left(Y_{0}\right)$. We first choose $f \in F$ so that $\varphi^{-1} f \varphi=a$. For each 
$t \in \varphi^{-1}\left(Y_{0}\right)$, there is a $g \in G_{0}$ satisfying $g \varphi(t) \in X-Y$. Then

$$
K\left(f g f^{-1}\right)=f(K(g)) \supset f \varphi(I)=\varphi a(I) \supset \varphi(I)
$$

implies that $f g f^{-1} \in G_{0}$. From

$$
f g f^{-1}(\varphi a(t))=f g f^{-1}(f \varphi(t))=f g \varphi(t) \in f(X-Y)=X-Y
$$

we infer that $a(t) \in \varphi^{-1}\left(Y_{0}\right)$ and $a \varphi^{-1}\left(Y_{0}\right) \subset \varphi^{-1}\left(Y_{0}\right)$. Since we can always find an $a \in A_{1}$ such that $a(I) \supset I$, and $a$ maps any point in $E^{1}-I$ into any other point further away from $I$, it follows that if $\varphi^{-1}\left(Y_{0}\right) \neq \varnothing$, then $\varphi^{-1}\left(Y_{0}\right)$ is the union of two half-lines, that is, $E^{1}-\varphi^{-1}\left(Y_{0}\right)=[\gamma, \delta] \supset[\alpha, \beta]=I$. We will show that $\varphi^{-1}\left(Y_{0}\right) \neq \varnothing$ and $[\alpha, \beta]=[\gamma, \delta]$ by deriving a contradiction from the assumption $\gamma\langle\alpha$. The case $\delta>\beta$ is handled in a similar manner. Let $C$ be the connected component of $\varphi^{-1}\left(K\left(g_{0}\right)\right)$ which contains [0,1]. Then $C$ is a closed interval with at least one endpoint $\varepsilon$, and we can find an $a_{0} \in A_{1}$ so that $a_{0}(C) \supset I$ and $a_{0}(\varepsilon)=\alpha$. If $f_{0} \in H(X)$ is an extension of $\varphi a_{0} \varphi^{-1}$, and $g_{1}=f_{0} g_{0} f_{0}^{-1}$, then

$$
K\left(g_{1}\right)=f_{0}\left(K\left(g_{0}\right)\right) \supset \varphi a_{0} \varphi^{-1}\left(K\left(g_{0}\right)\right) \supset \varphi a_{0}(C) \supset \varphi(I)
$$

implies that $g_{1} \in G_{0}$ and $a_{0}(C)$ is a connected component of $\varphi^{-1}\left(K\left(g_{1}\right)\right)$. Choose $y_{0} \in Y$ so that $g_{0}\left(y_{0}\right) \in X-Y$. From $g_{1}\left(f_{0}\left(y_{0}\right)\right)=f_{0} g_{0}\left(y_{0}\right) \in X-Y$ we infer that $Y_{0} \neq \varnothing$ and $\varphi^{-1}\left(Y_{0}\right) \neq \varnothing$. Evidently $t \in[\gamma, \alpha]$ implies $g_{1} \varphi(t) \in Y$, and we can find $t_{0} \in(\gamma, \alpha)$ so close to $\alpha$ that $t_{0} \neq$ $\varphi^{-1} g_{1} \varphi\left(t_{0}\right) \in(\gamma, \alpha)$. We may assume, in fact, that $\varphi^{-1} g_{1} \varphi\left(t_{0}\right)<t_{0}$; for if $\varphi^{-1} g_{1} \varphi\left(t_{0}\right)>t_{0}$, then we would work with $g_{1}^{-1}$. Choose $a_{1} \in A_{1}^{+}$so that $a_{1}(I) \supset I, a_{1}\left(t_{0}\right)=\gamma$, and let $f_{1} \in H(X)$ be an extension of $\varphi a_{1} \varphi^{-1}$. As we have already seen, $g_{2}=f_{1} g_{1} f_{1}^{-1} \in G_{0}$. Now

$$
\begin{aligned}
\varphi^{-1} g_{2} \varphi(\gamma) & =\varphi^{-1} f_{1} g_{1} f_{1}^{-1} \varphi(\gamma)=a_{1} \varphi^{-1} g_{1} \varphi a_{1}^{-1}(\gamma) \\
& =a_{1} \varphi^{-1} g_{1} \varphi\left(t_{0}\right)<a_{1}\left(t_{0}\right)=\gamma
\end{aligned}
$$

implies that $g_{2} \varphi(\gamma) \in Y_{0}$, and we can find $g_{3} \in G_{0}$ satisfying $g_{3}\left(g_{2} \varphi(\gamma)\right) \in$ $X-Y$. Since $g_{3} g_{2} \in G_{0}$, we conclude that $\varphi(\gamma) \in Y_{0}$ which contradicts our hypothesis. Hence, $\gamma=\alpha$, and our result is proved.

Theorem 12. Let $X$ be a topological space which can not be separated by any finite subset, $R$ a subgroup of $H(X), f \in H(X)$, $\varphi$ a homeomorphism from $E^{1}$ onto a closed subset $Y$ of $X$, and $S=\{g \in R: g(Y)=Y\}$. Suppose $\varphi^{-1} S \varphi \supset A_{1}, S_{0}=\{g \in G: Y \subset K(g)\}$ is 1-transitive on $X-Y, K(f) \supset \varphi([0,1])$, and $f(Y)-Y \neq \varnothing$. Then the group $G$ generated by $f$ and $R$ is $\omega$-transitive.

Proof. We proceed by induction on the transitivity and assume that $G$ is $n$-transitive for some $n \geqq 0$. If $x_{1}, \cdots, x_{n+1} \in X$ are given, 
$G_{0}=\left\{g \in G:\left\{x_{1}, \cdots, x_{n}\right\} \subset K(g)\right\}$, and we can show that $G_{0}\left(x_{n+1}\right)$ is an open subset of $X$, then Lemma 1 will imply that $G$ is $(n+1)$-transitive, and our induction step will be complete. By hypothesis, there is a $g_{0} \in G$ which maps $\left\{x_{2}, \cdots, x_{n}\right\}$ into $\varphi((0,1))$ and $x_{n+1}$ into $\varphi(1)$. We consider three cases for the position of $g_{0}\left(x_{1}\right)$. In case (i), $g_{0}\left(x_{1}\right) \in Y$ and $\varphi^{-1} g_{0}\left(x_{1}\right)<1$. Then we can find an interval $I=[\alpha, \beta]$ which contains $\varphi^{-1} g_{0}\left(x_{1}\right), \cdots, \varphi^{-1} g_{0}\left(x_{n}\right)$ but not $\varphi^{-1} g_{0}\left(x_{n+1}\right)$, and Lemma 10 gives us a $g_{1} \in G$ with the properties $\varphi(I) \subset K\left(g_{1}\right)$ and $g_{1}\left(g_{0}\left(x_{n+1}\right)\right) \in X-Y$. Since $S_{0}\left(g_{1} g_{0}\left(x_{n+1}\right)\right)=X-Y$ is open in $X$, it follows that

$$
g_{0}^{-1} g_{1}^{-1} S_{0} g_{1} g_{0}\left(x_{n+1}\right)=g_{0}^{-1} g_{1}^{-1}(X-Y)
$$

is open in $X$. From $g \in S_{0}$ we infer that

$$
K\left(g_{0}^{-1} g_{1}^{-1} g g_{1} g_{0}\right) \supset g_{0}^{-1} g_{1}^{-1}(Y) \supset g_{0}^{-1} g_{1}^{-1} \varphi(I)=g_{0}^{-1} \varphi(I) \supset\left\{x_{1}, \cdots, x_{n}\right\},
$$

whence $g_{0}^{-1} g_{1}^{-1} S_{0} g_{1} g_{0} \subset G_{0}$, and our induction step is complete in case (i). In case (ii), $g_{0}\left(x_{1}\right) \in X-Y$. Now Lemma 10 gives us a $g_{2} \in G$ with the properties $K\left(g_{2}\right) \supset\left\{g_{0}\left(x_{2}\right), \cdots, g_{0}\left(x_{n+1}\right)\right\}$ and $g_{2} \varphi(0) \in X-Y$. We can also find $g_{3} \in S_{0}$ satisfying $g_{3}\left(g_{2} \varphi(0)\right)=g_{0}\left(x_{1}\right)$. Setting $g_{4}=g_{2}^{-1} g_{3}^{-1} g_{0}$, we have

$$
\begin{array}{ll}
g_{4}\left(x_{i}\right)=g_{2}^{-1} g_{3}^{-1} g_{0}\left(x_{i}\right)=g_{0}\left(x_{i}\right), & 2 \leqq i \leqq n+1, \\
g_{4}\left(x_{1}\right)=g_{2}^{-1} g_{3}^{-1} g_{0}\left(x_{1}\right)=\varphi(0) . &
\end{array}
$$

Thus case (ii) can be reduced to case (i) with $g_{0}$ replaced by $g_{4}$. In case (iii), $g_{0}\left(x_{1}\right) \in Y$ and $\varphi^{-1} g_{0}\left(x_{1}\right)>1$. Again Lemma 10 gives us a $g_{5} \in G$ such that $K\left(g_{5}\right) \supset\left\{g_{0}\left(x_{2}\right), \cdots, g_{0}\left(x_{n+1}\right)\right\}$ and $g_{5}\left(g_{0}\left(x_{1}\right)\right) \in X-Y$. Setting $g_{6}=g_{5} g_{0}$, we have

$$
\begin{array}{ll}
g_{6}\left(x_{i}\right)=g_{5} g_{0}\left(x_{i}\right)=g_{0}\left(x_{i}\right), & 2 \leqq i \leqq n+1, \\
g_{6}\left(x_{1}\right)=g_{5} g_{0}\left(x_{1}\right) \in X-Y . &
\end{array}
$$

Thus case (iii) can be reduced to case (ii) with $g_{0}$ replaced by $g_{6}$, and all the cases relating to the position of $g_{0}\left(x_{1}\right)$ have been disposed of.

THEOREM 13. The conclusion of Theorem 12 remains valid if we replace $E^{1}$ by $\Pi^{1}$, that is, a circle, and $A_{1}$ by $P_{1}$.

Proof. The proof of Theorem 12 up to the definition of $g_{0}$ can be carried over unchanged. This time, however, we choose $g_{0}$ so as to map $\left\{x_{1}, \cdots, x_{n}\right\}$ into $\varphi((0,1))$ and consider two cases for the position of $g_{0}\left(x_{n+1}\right)$. In case (i), $g_{0}\left(x_{n+1}\right) \in X-Y$. As we have already seen in the proof of Theorem 12, this implies that $G_{0}\left(x_{n+1}\right)$ is open in $X$, and our induction step is complete in this case. In case (ii), $g_{0}\left(x_{n+1}\right) \in Y$. By hypothesis, these is some point $y_{0} \in Y-\varphi([0,1])$ 
satisfying $f\left(y_{0}\right) \in X-Y$. We choose $p_{1} \in P_{1}$ and a neighborhood $U$ of $\varphi^{-1} g_{0}\left(x_{n+1}\right)$ so that $U \subset \Pi^{1}-\left\{\varphi^{-1} g_{0}\left(x_{1}\right), \cdots, \varphi^{-1} g_{0}\left(x_{n}\right)\right\}, p_{1}\left(\varphi^{-1}\left(y_{0}\right)\right)=$ $\varphi^{-1} g_{0}\left(x_{n+1}\right)$, and $p_{1}\left(\Pi^{1}-[0,1]\right) \subset U$. Let $g_{1} \in S$ be an extension of $\varphi p_{1} \varphi^{-1}$, and $g_{2}=g_{1} f g_{1}^{-1}$. Then

$$
\begin{aligned}
K\left(g_{2}\right) & =g_{1}(K(f)) \supset g_{1} \varphi([0,1]) \\
& =\varphi p_{1}([0,1]) \supset \varphi\left(I^{1}-U\right) \supset\left\{g_{0}\left(x_{1}\right), \cdots, g_{0}\left(x_{n}\right)\right\}, \\
g_{2}\left(g_{0}\left(x_{n+1}\right)\right) & =g_{1} f g_{1}^{-1}\left(g_{0}\left(x_{n+1}\right)\right) \\
& =g_{1} f \varphi p_{1}^{-1} \varphi^{-1} g_{0}\left(x_{n+1}\right)=g_{1} f \varphi \varphi^{-1}\left(y_{0}\right) \in g_{1}(X-Y)=X-Y .
\end{aligned}
$$

If we set $g_{3}=g_{2} g_{0}$, then

$$
\begin{array}{rlrl}
g_{3}\left(x_{i}\right) & =g_{2} g_{0}\left(x_{i}\right)=g_{0}\left(x_{i}\right), & & 1 \leqq i \leqq n, \\
g_{3}\left(x_{n+1}\right) & =g_{2} g_{0}\left(x_{n+1}\right) \in X-Y, &
\end{array}
$$

and case (ii) can be reduced to case (i) with $g_{0}$ replaced by $g_{3}$. Thus all the cases relating to the positions of $g_{0}\left(x_{n+1}\right)$ have been disposed of.

CoRollary. Suppose $R$ is a subgroup of $H(X), f \in H(X), X \neq K(f)$ has a nonempty interior, and either (i) $X=E^{m}$ and $R=A_{m}$, or (ii) $X=\Pi^{m}$ and $R=P_{m}$. Then the group $G$ generated by $f$ and $R$ is $\omega$-transitive.

Proof. The case $m=1$ has already been verified in Theorems 8 and 10 , so we will assume that $m \geqq 2$. We first consider case (i) and choose points $x_{0} \in \operatorname{int} K(f)$ and $x_{1} \in E^{m}-K(f)$. If $f\left(x_{1}\right)$ does not lie on the line $Y$ through $x_{0}$ and $x_{1}$, then our result follows from Theorem 12, since $K(f) \cap Y$ contains a nondegenerate interval. If $f\left(x_{1}\right) \in Y$, then we choose a rotation $a_{1} \in A_{m}$ about the point $x_{1}$ through such a small positive angle that $K(f) \cap a_{1}^{-1}(Y)$ contains a nondegenerate interval I. Setting $f_{1}=a_{1} f a_{1}^{-1}$, we have

$$
\begin{aligned}
& K\left(f_{1}\right) \cap Y=a_{1}(K(f)) \cap Y=a_{1}\left(K(f) \cap a_{1}^{-1}(Y)\right) \supset a_{1}(I), \\
& f_{1}\left(x_{1}\right)=a_{1} f a_{1}^{-1}\left(x_{1}\right)=a_{1} f\left(x_{1}\right) \in X-Y,
\end{aligned}
$$

and our result again follows from Theorem 12 with $f$ replaced by $f_{1}$. Case (ii) is handled in exactly the same way, for we can identify $E^{m}$ with the finite part of $\Pi^{m}$, and $a_{1}$ can be extended to an element of $P_{m}$.

\section{REFERENCES}

1. Richard Arens, Topologies for homeomorphism groups, Amer. J. Math. 68 (1946), 593-610.

Received April 11, 1966.

UNIVERSITY OF BRITISH COLUMBIA

Vancouver, Canada 



\section{PACIFIC JOURNAL OF MATHEMATICS}

\section{EDITORS}

\section{H. ROYDEN}

Stanford University

Stanford, California

J. P. JANS

University of Washington

Seattle, Washington 98105

\section{J. DUGUNDJI}

Department of Mathematics

Rice University

Houston, Texas 77001

RICHARD ARENS

University of California

Los Angeles, California 90024

ASSOCIATE EDITORS

E. F. BECKENBACH

B. H. NeumanN

F. WoLF

K. YosidA

\section{SUPPORTING INSTITUTIONS}

\author{
UNIVERSITY OF BRITISH COLUMBIA \\ CALIFORNIA INSTITUTE OF TECHNOLOGY \\ UNIVERSITY OF CALIFORNIA \\ MONTANA STATE UNIVERSITY \\ UNIVERSITY OF NEVADA \\ NEW MEXICO STATE UNIVERSITY \\ OREGON STATE UNIVERSITY \\ UNIVERSITY OF OREGON \\ OSAKA UNIVERSITY \\ UNIVERSITY OF SOUTHERN CALIFORNIA
}

\author{
STANFORD UNIVERSITY \\ UNIVERSITY OF TOKYO \\ UNIVERSITY OF UTAH \\ WASHINGTON STATE UNIVERSITY \\ UNIVERSITY OF WASHINGTON \\ AMERICAN MATHEMATICAL SOCIETY \\ CHEVRON RESEARCH CORPORATION \\ TRW SYSTEMS \\ NAVAL ORDNANCE TEST STATION
}




\section{Pacific Journal of Mathematics}

\section{Vol. 23, No. 1 \\ March, 1967}

M. J. C. Baker, A spherical Helly-type theorem ................... 1

Robert Morgan Brooks, On locally m-convex*-algebras.............. 5

Lindsay Nathan Childs and Frank Rimi DeMeyer, On automorphisms of separable algebras ...................................

Charles L. Fefferman, A Radon-Nikodym theorem for finitely additive set

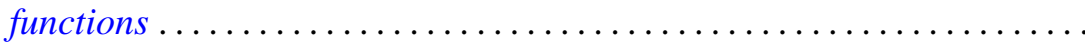

Magnus Giertz, On generalized elements with respect to linear

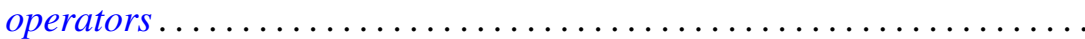

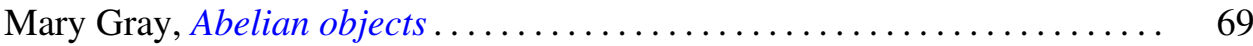

Mary Gray, Radical subcategories.............................. 79

John A. Hildebrant, On uniquely divisible semigroups on the two-cell . . . . . 91

Barry E. Johnson, AW*-algebras are $\mathrm{QW}^{*}$-algebras ............... 97

Carl W. Kohls, Decomposition spectra of rings of continuous functions . . . . 101

Calvin T. Long, Addition theorems for sets of integers .............. 107

Ralph David McWilliams, On $w^{*}$-sequential convergence and quasi-reflexivity ................................... 113

Alfred Richard Mitchell and Roger W. Mitchell, Disjoint basic



John Emanuel de Pillis, Linear transformations which preserve hermitian and positive semidefinite operators .

Qazi Ibadur Rahman and Q. G. Mohammad, Remarks on Schwarz's lemma

Neal Jules Rothman, An $L^{1}$ algebra for certain locally compact topological semigroups ...

F. Dennis Sentilles, Kernel representations of operators and their adjoints ...

D. R. Smart, Fixed points in a class of sets

K. Srinivasacharyulu, Topology of some Kähler manifolds

Francis C.Y. Tang, On uniqueness of generalized direct decompositions .

171 Albert Chapman Vosburg, On the relationship between Hausdorff dimension and metric dimension . 